\title{
Influence of water on the surface of graphene
}

\author{
Yunus Kaya ${ }^{1, a}$, Yalçın Kalkan ${ }^{2}$, and Rob Veenhof ${ }^{2,3}$ \\ ${ }^{1}$ Department of Chemistry, Faculty of Arts and Sciences, Uludag University, 16059 Bursa, Turkey \\ ${ }^{2}$ Department of Physics, Faculty of Arts and Sciences, Uludag University, 16059 Bursa, Turkey \\ ${ }^{3} R D 51$, CERN
}

\begin{abstract}
We have studied how water modifies the surface of graphene and in particular how the surface conductivity of graphene is affected. According to the literature, two types of interactions should be distinguished: physical, where a water molecule remains intact and is located at some distance from the mesh, and chemical, where a water molecule is imbricated in the graphene bond structure. We have developed theoretical models for both types of interactions using the density functional theory (DFT) with the B3LYP hybrid functional combined with the 6-31G(d) basis set. Our calculations show that the surface conductivity of graphene is reduced in the presence of water.
\end{abstract}

\section{Introduction}

Graphene, a two-dimensional sheet of $s p^{2}$ hybridised carbon atoms, is considered to be a next-generation electronic material due to its unique characteristics, such as high conductivity [1], optical transparency [2, 3], and ultrahigh mechanical strength [4]. Graphene has been investigated for applications in small and fast electronic devices, more efficient batteries, and stronger materials, amongst others. In addition, the exceptional surface properties of graphene hold promise for applications in biomaterials, medical instruments, microfluidic devices, sensors and energy devices [5, 6]. For example, a large hydrophilic electrode surface allows reactants to maximise the reaction surface area in aqueous systems for better electrocatalysis. The surface properties are governed by both chemical composition and geometrical structure, which can also significantly affect the performance of nano-materials [7].
Both experimental observations [8] and theoretical studies [9] have revealed that graphene is a strongly hydrophobic material, which limits its applications in contact deposition in solution and may contaminate the nano-electromechanical systems [10]. In addition, materials with controllable wetting properties are widely used in advanced multifunctional systems, such as biomaterials and microfluidic devices $[10,11]$. In particular, the wettability of graphene is essential for its utilisation in hybrid organic systems [12]. Although these hydrophobic properties, the graphene interacts with water molecules, and then the water may condense on the graphene changing its properties. In this study, we will examine how water modifies the surface conductivity of graphene.

\section{Computational methods}

All calculations were conducted using density functional theory (DFT) as implemented in the

ae-mail: ykaya@uludag.edu.tr 
GAUSSIAN 09 program package [13]. The molecular geometries and vibrational frequencies of all molecules in the ground state have been calculated using the B3LYP [14] hybrid functional with the 6-31G(d) basis set. Vibrational frequencies were used for calculation of the Gibbs free energies.

\section{Result and discussion}

Graphene may also find application in MPGDs, GEMs in particular. The hope is that a graphenecoated GEM lets electrons through but not ions. As a result, there would be no space charge due to ions drifting from their production point into the drift volume.

Graphene makes two types of interaction with water: physical and chemical. These interactions are shown in figure 1.

\begin{tabular}{|c|c|c|}
\hline Structure & $\begin{array}{c}\mathrm{H}_{2} \mathrm{O} \\
\text { Physically } \\
\text { absorbed }\end{array}$ & $\begin{array}{c}\mathrm{H}_{2} \mathrm{O} \\
\text { Chemically } \\
\text { absorbed }\end{array}$ \\
\hline & $a$ & \\
\hline 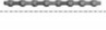 & 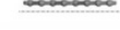 & \\
\hline
\end{tabular}

Figure 1. Atomic structures of a $\mathrm{H}_{2} \mathrm{O}$ molecule adsorbed on graphene with different types of defects.

In addition, there are four interaction orientations of the physical type. These orientation are shown in figure 2.

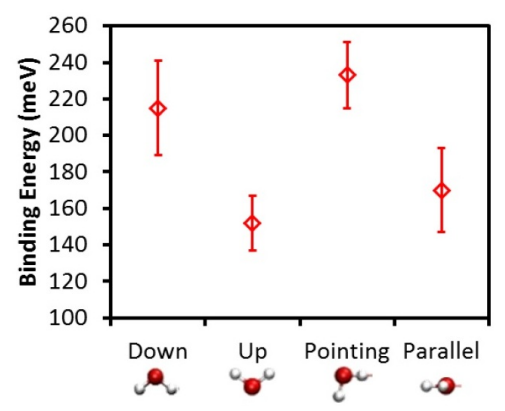

Figure 2. Various interaction orientations for water.
The binding energy is ca $180 \mathrm{meV}$ and has a weak water-orientation dependence.

Attempts to reproduce the gas phase data of graphene with an increasing number of rings showed that theoretical band gap tends to decrease. When the number of rings is 81 , the band gap decreases to $0.139 \mathrm{eV}$. This result is in agreement with experimental values, which are in the range $0-0.25 \mathrm{eV}$ [15]. All calculation results are shown in figure 3 . We have used a molecule with 81 rings for our calculations of graphene-water interactions.

The structure of the graphene sheet used in the present study are illustrated in figure 4. In the present study, we examined the physical and chemical interactions of a water molecule with graphene mesh as seen figure $4 \mathrm{~b}$ and figure $4 \mathrm{c}$. To determine the effect of water molecules on the surface conductivity of graphene, all modeled geometries were fully optimised at the DFT/6-31G(d) level.

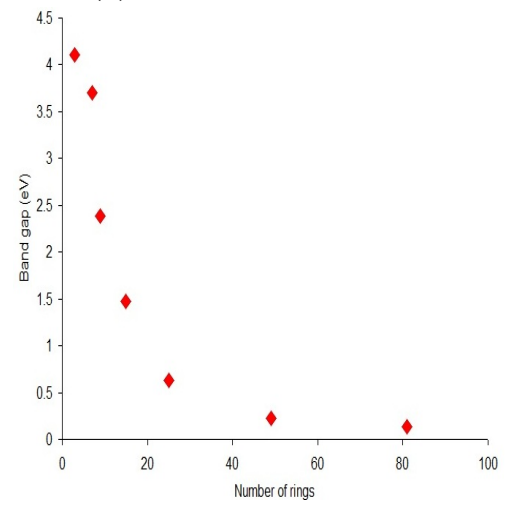

Figure 3. Theoretical band gap calculations of graphene through phenyl rings using 6-31G(d) basis set.

In addition, the band gaps for graphene, physically adsorbed graphene and chemically adsorbed graphene were calculated with the TDDFT method. The band gaps were used for explaining the conductivity. It was found that the band gap of graphene $0.139 \mathrm{eV}$. On the other hand, the band gaps were calculated at $0.143 \mathrm{eV}$ and $0.221 \mathrm{eV}$ for physically and chemically adsorbed water molecules, respectively. These results indicate that the band gap increases and 
the surface conductivity of graphene decreases in the presence of water molecules.
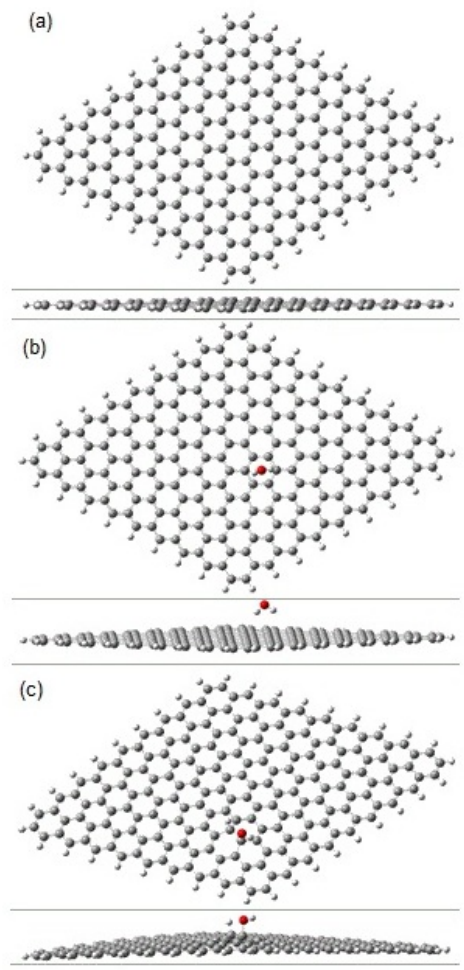

Figure 4. Optimised structures of molecules for 81 rings, a) graphene, b) physically adsorbed graphene and c) chemically adsorbed graphene.

\section{Conclusion}

Graphene has two types of interaction with water: physical and chemical. These interactions were studied theoretically with the DFT and TDDFT methods. All results indicate that the the surface conductivity of graphene decreases in the presence of water molecules.

\section{Acknowledgments}

This work is supported by the Scientific Research Projects Unit of Uludağ Univer- sity, project numbers: KUAP(F)-2015/20 and KUAP(F)-2013/25.

\section{References}

[1] Zhu, Y.F., Dai, Q.Q., Zhao, M., Jiang, Q. Sci. Rep. 3, 1524, (2013).

[2] Weber, C.M. et al., Small 6, 184-189 (2009).

[3] Yang, N., Zhai, J., Wang, D., Chen, Y., Jiang, L. ACS Nano 4, 887-894 (2010).

[4] Lee, C., Wei, X., Kysar, J. W., Hone, J. Science 321, 385-388 (2008).

[5] Abdelsayed, V. et al., J. Phys. Chem. Lett. 1, 2804-2809 (2010).

[6] Zhang, K., Dwivedi, V., Chi, C., Wu, J., J. Hazard. Mater. 182, 162-168 (2010).

[7] Feng, X. et al., J. Am. Chem. Soc. 126, 62-63 (2003).

[8] Wang, S., Zhang, Y., Abidi, N., Cabrales, L. Langmuir 25, 11078-11081 (2009).

[9] Leenaerts, O., Partoens, B., Peeters, F.M. Phys. Rev. B 79, 235440 (2009).

[10] Rafiee, J., Rafiee, M.A., Yu, Z.-Z., Koratkar, N. Adv. Mater. 22, 2151-2154 (2010).

[11] Chen, H., Müller, M.B., Gilmore, K.J., Wallace, G.G., Li, D. Adv. Mater. 20, 3557-3561 (2008).

[12] Bon, S. B., Piccinini, M.,Mariani, A., Kenny, J.M., Valentini, L. Diam. Relat. Mat. 20, 871-874 (2011).

[13] Frisch, M.J., et al., Gaussian 03, Revision E.01, Gaussian, Inc., Wallingford, CT, (2004).

[14] Becke, A.D., J. Chem. Phys. 98 56485652, (1993).

[15] Zhang, Y., Tang, Tsung-Ta, Girit, Caglar, Hao, Zhao, Martin, Michael C., Zettl, Alex, Crommie, Michael F., Shen, Y. Ron, Wang, Feng (11 June 2009). "Direct observation of a widely tunable bandgap in bilayer graphene". Nature 459 (7248): 820-823. 